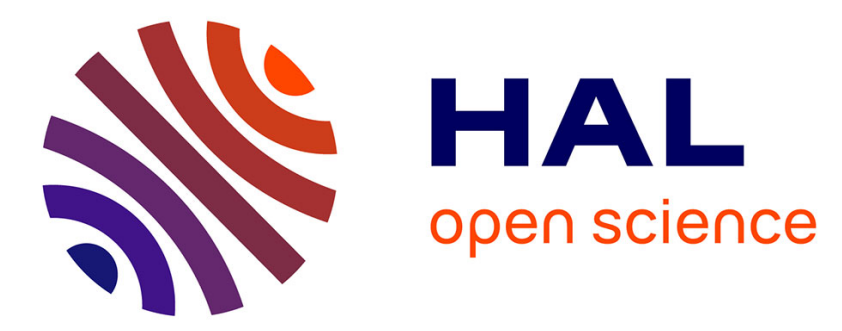

\title{
Versatile Encapsulation Technology Based on Tailored pH-Responsive Amphiphilic Polymers: Emulsion Gels and Capsules
}

Clémentine Locatelli-Champagne, Jean-Marc Suau, Olivier Guerret, Charlotte Pellet, Michel Cloitre

\section{To cite this version:}

Clémentine Locatelli-Champagne, Jean-Marc Suau, Olivier Guerret, Charlotte Pellet, Michel Cloitre. Versatile Encapsulation Technology Based on Tailored pH-Responsive Amphiphilic Polymers: Emulsion Gels and Capsules. Langmuir, 2017, 33 (49), pp.14020-14028. 10.1021/acs.langmuir.7b02689 . hal-02404907

\section{HAL Id: hal-02404907 https://hal.science/hal-02404907}

Submitted on 20 May 2021

HAL is a multi-disciplinary open access archive for the deposit and dissemination of scientific research documents, whether they are published or not. The documents may come from teaching and research institutions in France or abroad, or from public or private research centers.
L'archive ouverte pluridisciplinaire HAL, est destinée au dépôt et à la diffusion de documents scientifiques de niveau recherche, publiés ou non, émanant des établissements d'enseignement et de recherche français ou étrangers, des laboratoires publics ou privés. 


\title{
Versatile Encapsulation Technology Based on Tailored pH- Responsive Amphiphilic Polymers: Emulsion Gels and Capsules
}

\author{
Clémentine Locatelli-Champagne ${ }^{\dagger, \ddagger}$, Jean-Marc Suau ${ }^{\ddagger}$, Olivier Guerret ${ }^{\ddagger, \pm}$, Charlotte Pellet ${ }^{\dagger}$, and \\ Michel Cloitre ${ }^{\dagger}$ \\ ${ }^{\dagger}$ Soft Matter and Chemistry, CNRS, ESPCI Paris, PSL Research University, 10 rue Vauquelin, 75005 Paris (France) \\ ‡ Coatex SAS, 35 Rue Ampère, 69730 Genay (France) \\ \pm Mzi Life Sciences, 1 Rue Royale, 92210 Saint Cloud (France)
}

\begin{abstract}
tached to a methacrylic acid/ethylacrylate polyelectrolyte backbone. The composition and structure of the hydrophobic moieties of the side chains are customized in order to tune the particle morphology and the processing conditions. The technology exploits the synergy of properties provided by the copolymer: interfacial activity, $\mathrm{pH}$ responsiveness, and viscoelasticity. A one-pot process produces emulsion gels or capsule dispersions consisting of a hydrophobic liquid core surrounded by a polymer shell. The dispersions resist to high ionic strengths and exhibit long-term stability. The versatility of the method is demonstrated by encapsulating various hydrophobic substances covering a broad range of viscosities and polarities conventional and technical oils, perfumes, alkyd paints - with a high degree of morphological and rheological control.
\end{abstract}

ABSTRACT: We present a multipurpose technology to encapsulate hydrophobic substances in micron-size emulsion droplets and capsules. The encapsulating agent is a comb-like stimuli-responsive copolymer comprising side chain surfactants at-

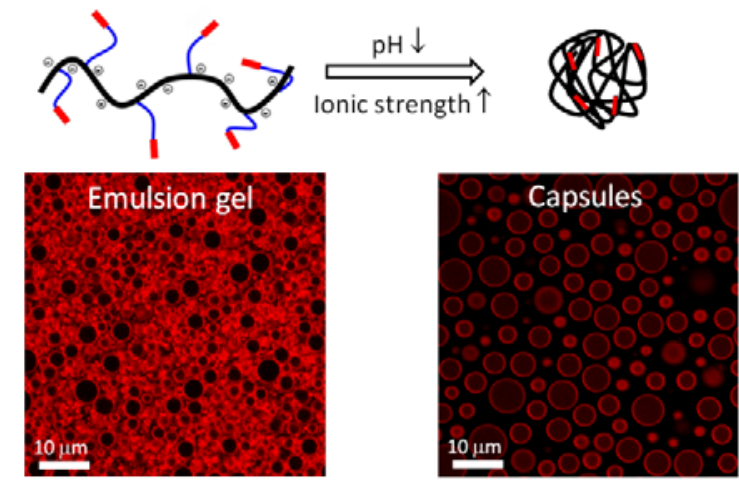

\section{INTRODUCTION}

Emulsions and capsules are fundamental components of modern liquid formulations used in pharmaceuticals, personal care products, food additives, and agricultural chemicals. $^{1-5}$ They have multiple functions such as to confine, transport, and release active substances, mask undesirable odor or taste, suppress volatile organic solvents in paints, and safely handle toxic or hazardous materials. Microcapsules can be produced by different methods: ${ }^{6}$ layer by layer deposition, ${ }^{7-12}$ polymerization or complexation at interfaces, ${ }^{13-16}$ emulsion templating, ${ }^{17,18}$ host-guest recognition, ${ }^{19,20}$ polymer phase separation, ${ }^{21,22}$ membrane emulsification, ${ }^{23}$ and microfluidics techniques. ${ }^{24,25}$ The great challenge in all these techniques is the choice of the encapsulating agent. Synthetic surfactants are widely available and easy to use but have important limitations, such as low efficiency, limited shelf-life stability, detrimental effects on the properties of final products, and possible toxicity for living organisms. Polymers, ${ }^{26}$ which can be block, ${ }^{27}$ comb-like, ${ }^{28}$ branched, ${ }^{29}$ star $^{30}$ polymers, circumvent many of these disadvantages and have great potential in applications. However the large-scale production of liquid capsules from polymeric additives is often hampered by the fact that it requires multi-step methods or presents limitations in terms of facile synthesis, availability, and versatility of the polymer for a wide range of applications.

Herein we describe a multipurpose polymer-based technology that allows one-pot fabrication, high encapsulation efficiency, versatility, environment friendliness, and industrial scale-up. We use functional comb-like copolymers consisting of side-chain surfactants attached to a polyelectrolyte backbone. They are synthesized by conventional radical polymerization and are customized by varying the composition and structure of the side chains. This specific architecture provides interfacial activity, viscoelasticity, and $\mathrm{pH}$-responsiveness. A one-pot process produces highly stable, micron-size emulsion droplets and capsules comprising a hydrophobic liquid core surrounded by a polymer shell. We demonstrate the versatility of the method by encapsulating a variety of hydrophobic substances covering a broad range of viscosities and polarities, with a high degree of morphological and rheological control, and long term stability. 


\section{MATERIALS AND METHODS}

Chemicals. Chemicals were purchased from SigmaAldrich (Silicon oil DC200), Fluka (fluorescent dyes Nile Red and Rhodamine B), and VWR (silicon oil Rhodorsil 47V20). Alkyd resin, Esterol A, and Citronellal were provided by Coatex (Arkema group). Olive oil was bought from groceries.

Synthesis and characterization of copolymers. The technology described in this paper is based on stimuli responsive copolymers which are designed and produced on demand. They have a comb-like architecture with a polyelectrolyte backbone and polyether side chains capped by hydrophobes, as shown in Table 1 . They are synthesized from ethyl acrylate $\left(\mathrm{EA} ; \mathrm{CH}_{2} \mathrm{CHCOOC}_{2} \mathrm{H}_{5}\right)$, methacrylic acid (MAA; $\mathrm{CH}_{2} \mathrm{CH}_{3} \mathrm{COOH}$ ) and alkyl-b-polyoxyethylene methacrylate macromonomers $\left[\mathrm{CH}_{2} \mathrm{CHCOO}\left(\mathrm{CH}_{2} \mathrm{CH}_{2} \mathrm{O}\right)_{n^{-}}\right.$ $\mathrm{X}]$. The backbone of the copolymers is composed of MAA, EA and the methacrylate end-groups of the macromonomers. The side-chains consist of a hydrophilic polyoxyethylene spacer $(n \cong 25-30)$ terminated by alkyl groups $\mathrm{X}$. The alkyl groups contain $m$ carbon atoms $(m=12,16)$ and have a linear $(\mathrm{L})$, branched $(\mathrm{B})$, or ramified $(\mathrm{R})$ architecture. The macromonomers derive from the surfactants $\left[\mathrm{HO}\left(\mathrm{CH}_{2} \mathrm{CH}_{2} \mathrm{O}\right)_{\mathrm{n}} \mathrm{X}\right]$, which result from ethoxylation of alcohols X-OH. In the following, the macromonomers and the surfactant precursors are designated by $\mathrm{M}(\mathrm{X}, m)$ and $\mathrm{S}(\mathrm{X}, m)$ where $\mathrm{X}=\mathrm{L}, \mathrm{B}, \mathrm{R}$, respectively, the copolymers by $\operatorname{PM}(\mathrm{X}, m)$, and the polymer backbones by $\mathrm{P}$.

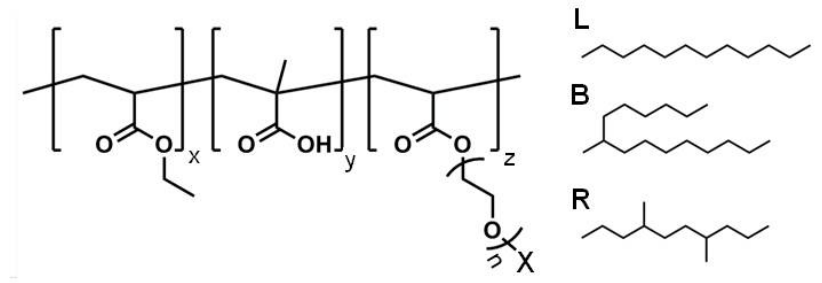

\begin{tabular}{ccccc} 
& $\mathrm{PM}(\mathrm{R}, 12)$ & $\mathrm{PM}(\mathrm{L}, 12)$ & $\mathrm{PM}(\mathrm{B}, 16)$ & $\mathrm{PM}(\mathrm{L}, 16)$ \\
\hline \hline$x$ & 50.2 & 50.0 & 50.4 & 52.3 \\
$y$ & 49.3 & 49.3 & 49.0 & 47.1 \\
$\mathrm{z}$ & 0.5 & 0.7 & 0.6 & 0.7 \\
$\Gamma_{\infty}$ & 7.0 & 9.2 & 8.1 & 10.9 \\
$(\mathrm{mN} / \mathrm{m})$ & & & & \\
$c a c(\mathrm{~g} / \mathrm{g})$ & $7 \times 10^{-3}$ & $2 \times 10^{-3}$ & $10^{-3}$ & $9 \times 10^{-4}$ \\
{$[\mathrm{M}]_{\mathrm{cac}}$} & $3.5 \times 10^{-4}$ & $1.4 \times 10^{-4}$ & $6.0 \times 10^{-5}$ & $6.3 \times 10^{-4}$ \\
$(\mathrm{M})$ & & & & \\
\hline \hline
\end{tabular}

Table 1. Chemical structure, composition and solution properties of copolymers $\mathrm{PM}(\mathrm{X}, m)$. The alkyl groups $\mathrm{X}$ carried by the side chains can be: $\mathrm{L}(m=12,16), \mathrm{B}$ $(m=16)$, and $\mathrm{R}(m=12), x, y, z$ are expressed in mole $\%$. $\Gamma_{\infty}$ is the interfacial tension between the copolymers solutions and silicone oil at large concentration; cac is the critical aggregation concentration; $\left([\mathrm{M}]_{\mathrm{cac}}\right.$ is the effective concentration of macromonomers at the cac. The interfacial tension of the polymer $\mathrm{P}$ without pendant groups is $\Gamma_{\infty}=6.1 \mathrm{mN} / \mathrm{m}$.
The copolymers $\mathrm{PM}(\mathrm{X}, \mathrm{m})$ were synthesized at low $\mathrm{pH}$ $(\cong 2)$ in latex form from EA, MAA, and macromomers $\mathrm{M}(\mathrm{X}, m)$ using semi-continuous emulsion polymerization under starved feed conditions. Linear chains $\mathrm{P}$ identical to the backbones of $\mathrm{M}(\mathrm{X}, m)$ were synthesized from ethyl acrylate and methacrylic acid in the same conditions. The polymer latexes were dialyzed in cellulose tubular membranes for approximately one month prior to analysis. The copolymers have a collapsed conformation at low $\mathrm{pH}$ but swell and become soluble upon neutralization of the carboxylic functions of MAA by a base. All experiments reported in the following were conducted after complete neutralization with $1 \mathrm{M} \mathrm{NaOH}$. The molecular weight of the linear chains $\mathrm{P}$ was determined by size exclusion chromatography in $\mathrm{LiBr} 0.5 \mathrm{M}$ solvent (Viscotek TDA 302), using a polyethylene oxide standard: $M_{\mathrm{n}} \cong 2 \times 10^{5} \mathrm{~g} / \mathrm{mol}$ with a polydispersity index of about 2.6. The compositions of the amphiphilic copolymers were determined by $1 \mathrm{H}$ NMR using a Bruker Avance 400 spectrometer (Table 1).

Interfacial properties of copolymer solutions. The interfacial tension between aqueous solutions of fully ionized copolymers and silicon oil was measured by the pendant drop technique using a home-made set-up. Pendant drops hanging from a needle tip in a silicone oil bath were imaged at high magnification using a CCD camera. Images were recorded at regular time intervals and the drop shapes were fitted to the mathematical expression of the profile where the interfacial tension is an adjustable parameter.31 All data reported in the following were measured at equilibrium. The interfacial tension decreases with the concentration before plateauing at a constant value $\Gamma_{\infty}$ (Figure S1). Table 1 shows that the value of $\Gamma_{\infty}$ depends on the number $m$ of carbons and the architecture of the alkyl hydrophobes.

Associative properties of copolymers solutions. The alkyl groups of the copolymers $\mathrm{PM}(\mathrm{X}, m)$ form hydrophobic domains above the critical aggregation concentration (cac).$^{32}$ We determined the cac of the different copolymers using Nile Red fluorescence. Nile Red is a solvatochromic dye which fluoresces in hydrophobic environments. ${ }^{33}$ Samples of increasing concentration were prepared by adding small aliquots of a stock solution of Nile Red in ethanol $\left(C_{\mathrm{NR}}=2.5 \times 10^{-3} \mathrm{M}\right)$ to copolymer solutions $\left(c_{\mathrm{NR}}=2 \times 10^{-6} \mathrm{M}\right)$. After vigorous stirring the samples were kept at rest and in the dark during 24 hours prior to further investigation. The fluorescence properties were measured with a spectrofluorophotometer from Shimadzu Corporation (RF-5301PC) using an excitation wavelength of 543 $\mathrm{nm}$. Emission spectra were recorded from $575 \mathrm{~nm}$ to 800 $\mathrm{nm}$ at $1-\mathrm{nm}$ interval. The photoreceptor is placed at $90{ }^{\circ} \mathrm{C}$ from the incident beam. The total fluorescence intensity was obtained by integration of the signal over the entire wavelength window after subtraction of the baseline. The intensity is negligible at low concentrations but rapidly increases above a well-defined concentration which is interpreted as the critical aggregation concentration (Figure S1). ${ }^{33}$ The cac correlates to the onset of the interfacial en- 
ergy plateau found at large concentration as for low molecular weight surfactants. In Table 1, we report the values of the cac measured for the copolymers $\operatorname{PM}(X, m)$ as well as the effective concentration of the macromonomer at the cac, $[\mathrm{M}]_{\mathrm{cac}}$. The latter is very low and compares very well with the critical micellar concentration of the free surfactants $\mathrm{S}(\mathrm{X}, \mathrm{m})$ which was determined independently (data not shown).

Rheological properties of copolymer solutions. Due to the ability of the side-chain groups to aggregate into micelles, copolymers $\mathrm{PM}(\mathrm{X}, m)$ are associative polymers which form transient networks in solution. ${ }^{34}$ We characterized the nonlinear viscoelastic properties of the copolymer solutions above the cac using a MCR 501 rheometer from Anton Paar equipped with a cone and plate geometry (diameter: $50 \mathrm{~mm}$; angle: $2^{\circ}$ ). The viscoelastic properties depend on the architecture of the alkyl groups $\mathrm{X}$ (Figure S2): (i) for a given number $m$ of carbon atoms, both the viscosity and the normal stress differences are higher for linear alkyl groups than for branched or ramified groups; (ii) for the linear architecture, the viscosity and the normal stresses increase drastically with $m$. Solutions of copolymer $\operatorname{PM}(\mathrm{L}, 16)$ are very viscous and develop large normal stresses even at low concentration.

Characterization of particles. Emulsion gels and capsule dispersions were stained before observation with Nile Red or Rhodamine B at a concentration of $10^{-6} \mathrm{M}$. Nile Red reveals the hydrophobic domains of the copolymer when it is illuminated at $\lambda_{\text {exc }}=543 \mathrm{~nm}$ (confocal images are red in the following) or the oil droplets when $\lambda_{\text {exc }}=477 \mathrm{~nm}$ (green images); ${ }^{33}$ Rhodamine B stains uniformly the continuous aqueous phase when $\lambda_{\mathrm{exc}}=514 \mathrm{~nm}$ (green images). After staining, the dispersions were deposited on a disposable cuvette slide covered with a thin coverslip. Confocal imaging was performed using a Zeiss LSM 510 laser scanning microscope equipped with oil immersion objectives (Zeiss Plan-Apochromat 63×, NA=1.4; Zeiss Plan-Neofluar $40 \times, \mathrm{NA}=1.3)$. For each sample, 10 full frame images $(512 \times 512$ pixels $)$ containing a total of about 1500 particles were recorded and analyzed. Each series of images was processed using a home-built ImageJ macro which successively performs the following operations: image binarization, particle contour extraction, particle cross-sectional area calculation, and equivalent diameter determination. Details smaller than 2 pixels were excluded from the statistics. The particle size distributions based on 2D images are affected by some uncertainly since the plane of observation intersects the particles along different meridians, resulting in underestimations of the actual size of the particles. This effect was corrected using an algorithm based on the Saltykov method which computes 3D particle size distributions. ${ }^{35}$ The distributions were analyzed in terms of relative and cumulative volume weighted distributions. The particle size was characterized by the volume mean diameter $\left.D[4,3]=\sum d_{\mathrm{i}}^{4} / \sum d_{\mathrm{i}}^{3}\right)$ and the volume median diameter $d(v, 0.5)$ for which the cumulative undersized volume fraction is equal to 0.50 . The uniformity factor $U=\sum d_{\mathrm{i}}^{3}\left[d_{\mathrm{i}}-d(v, 0.5)\right] /\left[d(v, 0.5) \sum d_{\mathrm{i}}^{3}\right]$ parametrizes the polydispersity. A dispersion is usually considered as monodisperse when $U$ is smaller than $0.25 .^{36}$

Diffusive Wave-Spectroscopy (DWS). DWS was used to characterize the aging properties of highly turbid emulsion gels and capsule suspensions. DWS measurements were performed at $20{ }^{\circ} \mathrm{C}$ using a home-made set-up working in transmission geometry. The samples were introduced in glass cuvettes having an optical path length of $10 \mathrm{~mm}$, which were uniformly illuminated by a $1-\mathrm{cm}-$ diameter laser beam. The scattered intensity was collected by a monomode optical fiber connected to a photomultiplier (EMI) and analyzed by a digital correlator (Brookhaven BI9000) which computes the time averaged intensity $I$ and the intensity autocorrelation function $g_{2}(t)$. The propagation of light in the multiple scattering limit can be described as a diffusive process where each photon is scattered a very large number of times and follows a random walk with an elementary step corresponding to the transport mean free path $\ell^{*}$. The value of $\ell^{*}$ is extremely sensitive to the geometry, size, refractive index and concentration of the scatterers, so that it can be used to characterize the properties of a time-evolving system. ${ }^{37}$ To determine $\ell^{*}$, we measured the transmittance through a slab of thickness $L$ of the unknown sample $(T)$ relative to a reference sample $(\widetilde{T})$ of known $\widetilde{\ell^{*}} \cdot{ }^{37}$ The ratio $T / \tilde{T}$ yields the unknown transport mean path $\ell^{*}: 38$

$$
\frac{T}{\tilde{T}}=\frac{\ell^{*}}{\ell^{*}} \frac{3 L+4 \widetilde{\ell^{*}}}{3 L+4 \ell^{*}}
$$

The reference sample was a suspension of calibrated polystyrene beads from Duke Scientific Corporation (diameter: $160 \mathrm{~nm}$ ), which has a transport mean free path calculated from Mie theory equal to $\widetilde{\ell^{*}}=0.125 \pm 0.005 \mathrm{~mm}$.

\section{RESULTS}

Preparation of emulsion gels and capsules. We use the one-pot method described in Figure 1. The polymer in latex form is introduced with sodium hydroxide and water into a flask at a concentration larger than the critical aggregation concentration $\left(10^{-2} \mathrm{~g} / \mathrm{g}<\mathrm{C}<4 \times 10^{-2} \mathrm{~g} / \mathrm{g}\right)$. Upon ionization of the MAA monomers, a homogeneous viscoelastic solution at $\mathrm{pH} \cong 8.5-9$ is obtained (Figure 1a). The hydrophobic phase, termed the oil in the following, is added to the viscoelastic polymer solution under moderate stirring using a Heidolph RZR1 mixer (Figure 1b). Very viscous liquids such as alkyd resins can be emulsified but they have to be pre-heated at $40^{\circ} \mathrm{C}$. The final volume fraction of the dispersed oil phase is $0.05<\phi<0.75$. After complete incorporation, we obtain an emulsion gel made of micron-size oil droplets interconnected through a copolymer network bridged by micelles of the associating groups $\mathrm{X}$ (Figures 1b-b'). Because of the comb-like structure of the copolymers a great number of hydrophobic groups ( $\cong 25$ per chain) are solubilised in the hydrophobic core of the droplets. This structure imparts yielding properties to the emulsion gel. The yield stress depends on the polymer concentration, oil volume fraction, emulsion droplet size, and associative group architecture. Liquid capsules are obtained by adding an acid under continuous stirring to the 


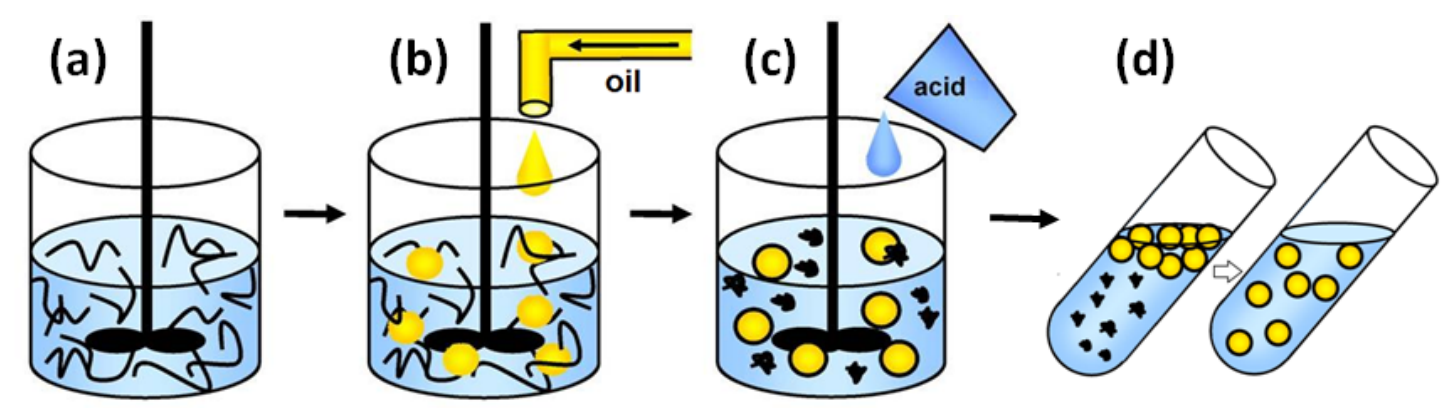



$\left(b^{\prime}\right)$
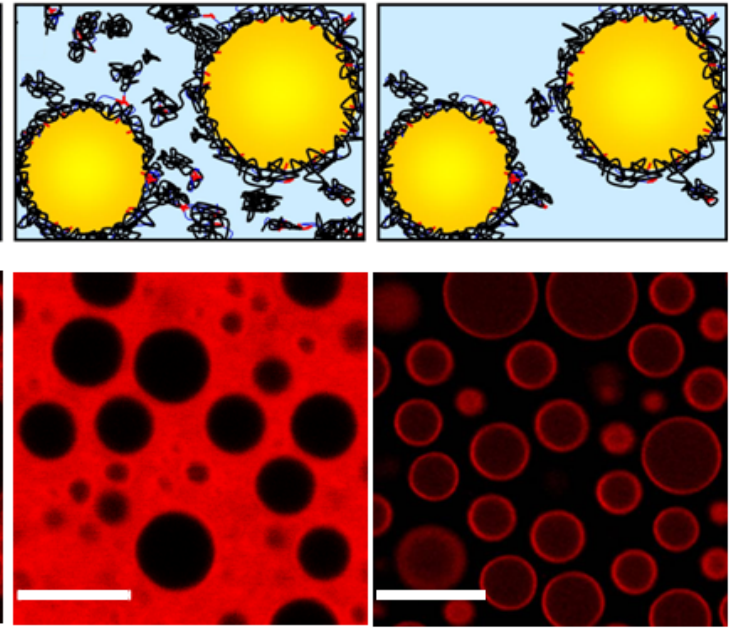

$\left(c^{\prime}\right)$

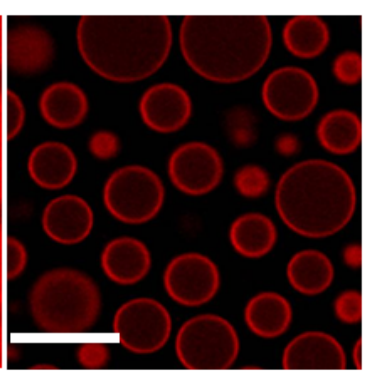

$\left(d^{\prime}\right)$

Figure 1. Manufacture of emulsion gels and capsules. The top diagrams depict the four steps of the process: polymer dissolution (a), emulsion gel formation upon silicon oil addition (b), encapsulation upon acid addition (c) removal of excess polymer (d). The confocal microscopy images at the bottom show an emulsion gel at $\mathrm{pH}=8.5$ (b'), a capsule dispersion before (c') and after (d') removal of the excess polymer $\left(\mathrm{pH}=5\right.$ and 7 respectively). The dye is Nile Red $\left(\lambda_{\text {exc }}=543 \mathrm{~nm}\right)$. Fluorescence is weak in the continuous phase of ( $b^{\prime}$ ) because the polymer is soluble at $\mathrm{pH}=8.5$. Large fluorescence in the continuous phase of (c') and at the droplet surfaces in (d') reveals apolar environments. The scale bar represents $5 \mu \mathrm{m}$. The copolymer is $\mathrm{PM}(\mathrm{B}, 16)$ at $C=0.033 \mathrm{~g} / \mathrm{g}$; the oil viscosity is $\eta_{\mathrm{d}}=0.52 \mathrm{~Pa} . \mathrm{s}$.

emulsion gel; here we used phosphoric acid. Upon acidification, the macromolecules adsorbed at the oil/water interface collapse into a thin polymeric shell covering the droplets whereas the inter-droplet network precipitates into the continuous phase (Figure 1c,c'). We end up with a dispersion of capsules consisting of a liquid core stabilized by a layer of insoluble polymer. The excess polymer in the aqueous phase can be eliminated on demand. After the capsule suspension is centrifuged for a few minutes at moderate acceleration, the capsules accumulate at the top of the tube. The liquid phase underneath, which contains the excess polymer, is removed. The capsules are then redispersed under gentle agitation in pure water with neutral $\mathrm{pH}$. This treatment does not provoke the coalescence of the capsules during centrifugation or redispersion. Generally three successive centrifugal clean up cycles suffice to eliminate the excess polymer from the aqueous phase (Figure $1 \mathrm{~d}, \mathrm{~d}$ ').

To relate the particle size to the shearing conditions and copolymers properties, capsules were manufactured under well-defined shearing conditions. A premix of oil and copolymer solution was loaded in the cone and plate or double Couette geometry of a commercial rheometer Anton
Paar MCR501, and sheared at imposed shear rate.

Fingerprinting capsule formation. To fingerprint the formation of capsules, we measured the effective viscosity and volume mean diameter of droplets during acidif-

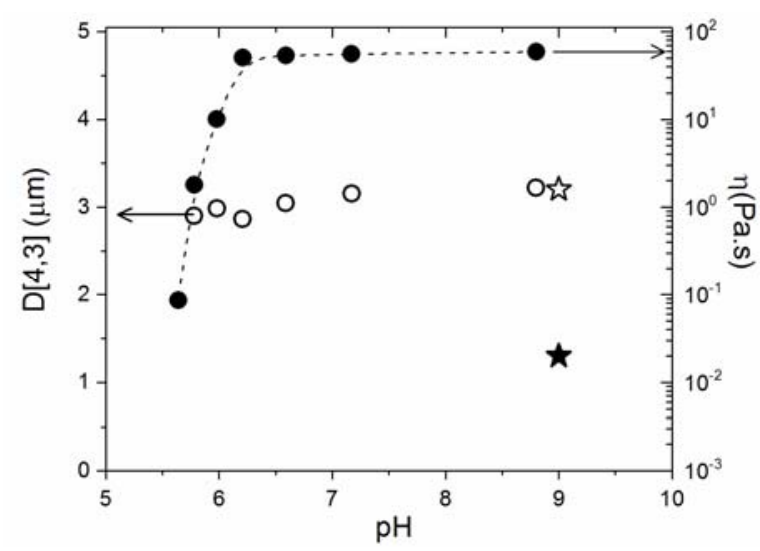

Figure 2. Volume mean diameter of particles $(O, \downarrow)$ and effective viscosity $(\boldsymbol{\bullet}, \star)$ during encapsulation of silicon oil ( $\phi=0.40 ; \eta_{\mathrm{d}}=0.52$ Pa.s) using $\mathrm{PM}(\mathrm{B}, 16)$ at $C=0.033$ $\mathrm{g} / \mathrm{g}$. Star symbols refer to samples containing $1 \mathrm{M} \mathrm{NaCl}$. 

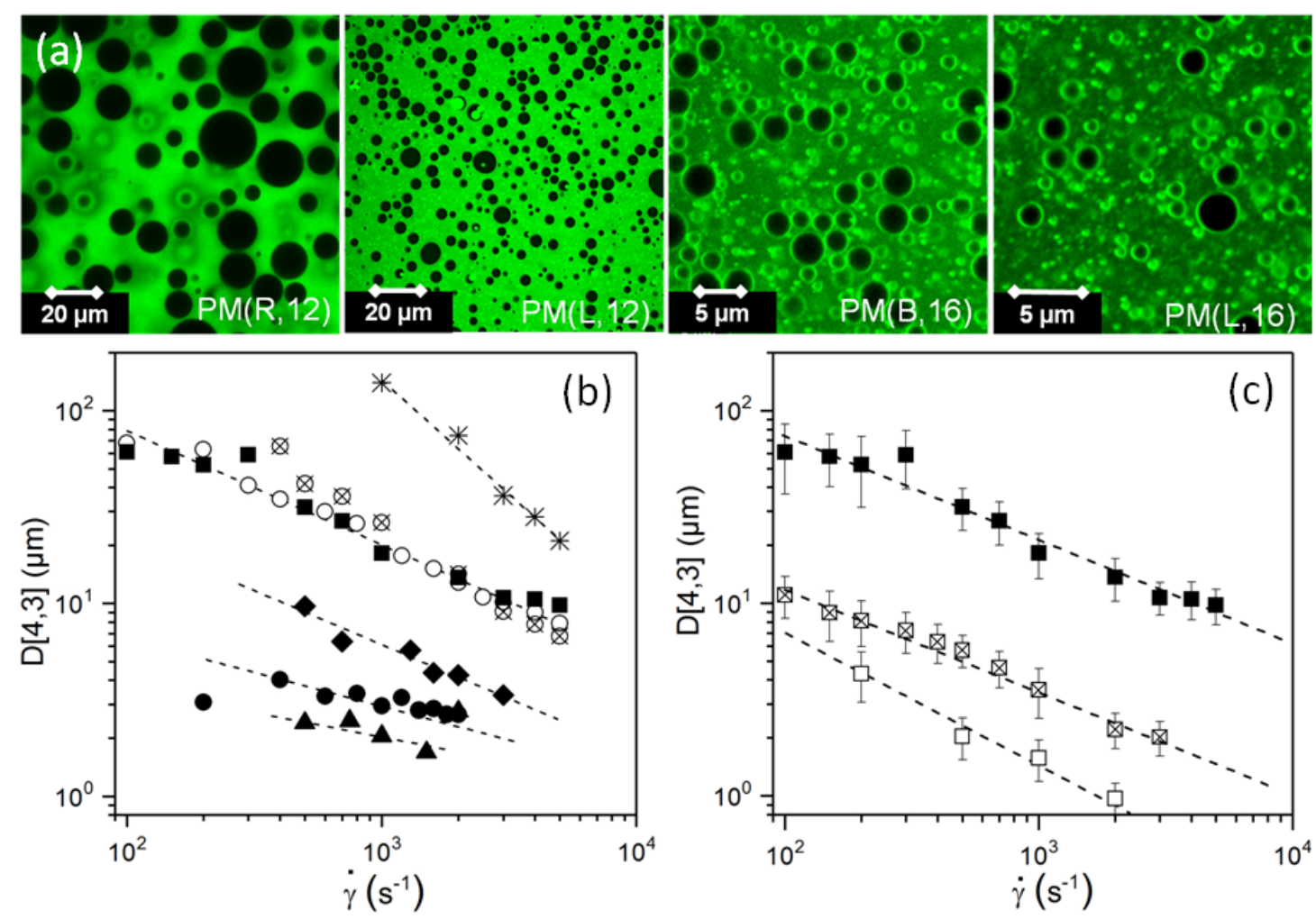

Figure 3. (a): confocal microscopy images of silicone oil/water emulsion gels prepared with different copolymers at the same polymer concentration $C=0.033 \mathrm{~g} / \mathrm{g}$ and same shear rate $\dot{\gamma}=500 \mathrm{~s}^{-1}$. The dye is Rhodamine B which stains the continuous aqueous phase $\left(\lambda_{\mathrm{exc}}=514 \mathrm{~nm}\right)$. (b): variations of the droplet volume mean diameter of droplets versus the applied shear rate for a series of copolymers at the same concentration $C=0.033 \mathrm{~g} / \mathrm{g}[\mathrm{P}: \bigcirc ; \mathrm{PM}(\mathrm{R}, 12): \mathbf{\square} ; \mathrm{PM}(\mathrm{L}, 12): \bullet$; $\mathrm{PM}(\mathrm{B}, 16):-\mathrm{O} M(\mathrm{~L}, 16): \boldsymbol{\Delta}]$, the surfactant $\mathrm{S}(\mathrm{B}, 16)$ at $C=0.17 \mathrm{~g} / \mathrm{g}(*)$, and a mixture $(\otimes)$ of $\mathrm{P}$ at $C=0.033 \mathrm{~g} / \mathrm{g}$ and $\mathrm{S}(\mathrm{B}, 16)$ at $C=0.033 \mathrm{~g} / \mathrm{g}$. (c): variations of the volume mean diameter of droplets versus the applied shear rate for copolymer $\operatorname{PM}(\mathrm{R}, 12)$ at $C=0.033 \mathrm{~g} / \mathrm{g}(\mathbf{\square}), \mathrm{C}=0.066 \mathrm{~g} / \mathrm{g}(\bigotimes)$, and $\mathrm{C}=0.10 \mathrm{~g} / \mathrm{g}(\square)$. The viscosity of the oil is $\eta_{\mathrm{d}}=0.52 \mathrm{~Pa} . \mathrm{s}$ and the volume fraction is $\phi=0.40$.

ication (Figure 2). The particle size does not change with the $\mathrm{pH}$. For $\mathrm{pH}>6.5$, the effective viscosity is constant indicating that the emulsion gel structure persists. Below $\mathrm{pH} \cong 6.2$, the copolymer network collapses causing a decrease of the continuous phase viscosity and a drop of the effective viscosity of the dispersion by several orders of magnitude. At $\mathrm{pH} \cong 4$ (data not shown), the electrostatic repulsion associated with the negative charges borne by the copolymers no longer stabilizes the droplets against aggregation. Clusters of droplets form and cream at the top of the dispersion but they are reversibly dispersed upon addition of sodium hydroxide. The insoluble polymeric shells wrapping the particles stabilize the particles against coalescence. We never observed destabilization, particle rupture, or oil leakage during and after encapsulation. Capsules can also be obtained by adding sodium chloride which increases the ionic strength and provokes the collapse of the copolymer (Figure 2). These results demonstrate that the capsule size is set during emulsification. Most of the data presented in the following concern droplets in particle gels but they directly extrapolate to capsules. From the results shown in Figure 2, we see that the rheological properties of the final dispersions can be adjusted on demand independently of the particle size by tuning the $\mathrm{pH}$ and the ionic strength without any addition of rheological additives.

Influence of processing conditions and copolymer on particle formation. Figure 3 explores the relation between polymer architecture, concentration, processing conditions, and particle size. Figure $3 \mathrm{a}$ shows that well defined particles are obtained with all the copolymers that are available. However when the concentration and the shear rate are fixed, the particle size is strongly dependent on the copolymer as we will now discuss. Figure $3 \mathrm{~b}$ shows the variations of the volume mean diameter $\mathrm{D}[4,3]$ with the applied shear rate for different surfactant systems. We observe that $\mathrm{D}[4,3]$ decreases with increasing shear rate as power laws. The exponent is not universal and depends on the copolymer. Figure $3 \mathrm{~b}$ highlights several important results. First, the comb-like architecture of the copolymers provides a remarkable synergistic effect: copolymer $\operatorname{PM}(\mathrm{B}, 16)$ produces much smaller particles than surfactant $\mathrm{S}(\mathrm{B}, 16)$ alone at a much larger concentration, and mixtures of $S(B, 16)$ with the polymer backbone $P$ without pendant groups. Secondly, the volume mean diameter $\mathrm{D}[4,3]$ of the droplets and capsules sensitively depends on the number of carbon atoms $(m)$ and the architecture of the hydrophobic groups $X$. For a given architecture, D[4,3] decreases when $m$ increases. At constant $m$, the functional role of the 
groups X can be ranked in increasing order of particle diameter according to linear $<$ branched $<$ ramified. The droplets formed by the unmodified polymer $\mathrm{P}$ are significantly larger.

Let us now investigate how the polymer concentration of the aqueous phase affects the droplet size. Figure $3 \mathrm{c}$ shows the variations with the shear rate of the volume mean diameter for copolymer $\mathrm{PM}(\mathrm{R}, 12)$ at different concentrations. Similar results are obtained with the other copolymers. Again we find that the droplet size decreases with the applied shear rate as power laws; the new result here is that the exponent strongly depends on the polymer concentration. We also observe that increasing the polymer concentration leads to a drastic reduction of the particle diameter.

In Figure 3, micron-size particles are readily obtained at shear rates as low as a few $100 \mathrm{~s}^{-1}$ in particular when the linear PM(L,12) and PM(L,16) copolymers or the branched $\mathrm{PM}(\mathrm{B}, 16)$ copolymer are used. Smaller particles could be made by increasing the shear rate using a continuous high shear Couette mixer. ${ }^{36}$ We performed a systematic study of the particle diameter for different shearing times and found that steady state values are reached after periods of time as short as $10 \mathrm{~s}$.

Influence of the dispersed phase. The dispersed phase is another important parameter. The technology is remarkably insensitive to the oil volume fraction, $\phi$. We were equally successful in preparing very dilute $(\phi \cong 0.05)$ and highly concentrated dispersions of droplets and capsules $(\phi \cong 0.75)$. The particle size is constant apart from a slight decrease when the oil volume fraction $\phi$ increases up to the jammed regime (Figure S3).

A second important parameter is the viscosity contrast between the continuous and dispersed phases. We were able to encapsulate oils which are much more viscous than to the continuous phase (Figure S4). The results can be rationalized in terms of the importance of the viscosity ratio $p=\eta_{\mathrm{d}} / \eta_{\mathrm{c}}$ where $\eta_{\mathrm{c}}$ and $\eta_{\mathrm{d}}$ are the viscosities of the dispersed and continuous phases respectively. Here $\eta_{\mathrm{c}}$ is a function of the shear rate. The smallest particle sizes are obtained when $p$ is of the order of 1 . When the viscosities are not matched $(p \neq 1)$, larger particles are obtained. This is reminiscent of conventional emulsification of Newtonian liquids where particle formation is not possible when the viscosity of the dispersed phase is too small or exceeds several times the viscosity of the continuous phase. ${ }^{39}$

Polydispersity of droplets and capsules. In many applications one important question beyond the mean particle size concerns the size distribution and in particular the possibility to obtain narrow size distributions. We systematically analyzed the confocal microscopy images and we determined particle size distributions using the protocol presented in the experimental section. Volume weighted relative and cumulative particle size distributions are shown in Figure 4 for silicon oil capsule dispersions prepared with polymer PM(L,12). Similar results are obtained with the other oils with very little sensitivity to preparation conditions. The polydispersity of the dispersions is charac-

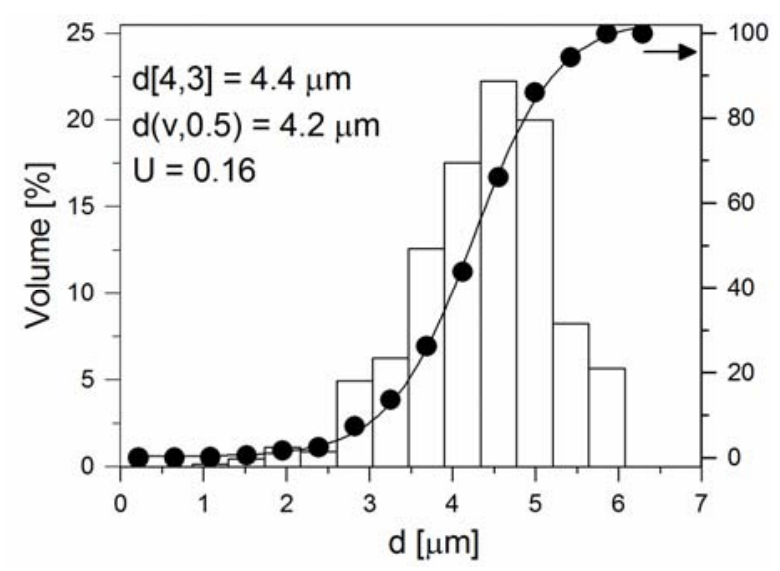

Figure 4. Volume weighted relative and cumulative particle size distributions (left and right hand side axis respectively) for silicon oil in water capsules prepared with $\operatorname{PM}(\mathrm{L}, 12)$ at $\mathrm{C}=0.033 \mathrm{~g} / \mathrm{g} \quad\left(\phi=0.40 ; \eta_{\mathrm{d}}=0.52\right.$ Pa.s; $\dot{\gamma}=1600 \mathrm{~s}^{-1}$ )

terized by the uniformity parameter $U$, which is always found smaller than 0.25 . This indicates that the dispersions can be considered as fairly monodisperse. ${ }^{36}$

Long-term stability of emulsion gels and capsules. Emulsion gels and capsules can be stored for months without apparent of signs of coalescence, coarsening, or oil release (Figure S5). The long-term stability can be quantitatively evaluated using direct confocal microscopy observations combined with the highly sensitive DWS technique. Emulsion gels and capsules are studied as prepared, the continuous phase being an associative polymer solution and water, respectively.

First we measured the transport mean free path of emulsion gels and capsules suspensions with different particle sizes. The transport mean free path $\ell^{*}$ measured by DWS was found to be related to the particle diameter $D$ measured by confocal microcopy through the approximate expression: $\ell^{*} \cong 166+41 D$. The experimental accuracy on $\ell^{*}$ values being of the order of $10 \mu \mathrm{m}$, the technique appears to be a sensitive indicator of the particle size evolution. For the three sets of data presented in Figure 5a, we find that the transport mean free paths of emulsion gels and capsules remain constant over the duration of the experiments, here 14 days. The data for the capsules with the largest mean free path are slightly scattered because the droplets are larger and the continuous phase (water) has a low viscosity, so that they tend to cream and create volume fraction inhomogeneities. From these data we conclude that the particle size of emulsion gel droplets and capsules do not change over weeks. The long term stability of capsules dispersions can also been evaluated from the dynamic properties of the dispersions by measuring intensity correlation functions at different times after preparation. Figure $5 b$ shows results obtained for a concentrated capsule suspension. The shapes of the correlation functions and the decay times remain exactly the same over the longest periods of time investigated. 

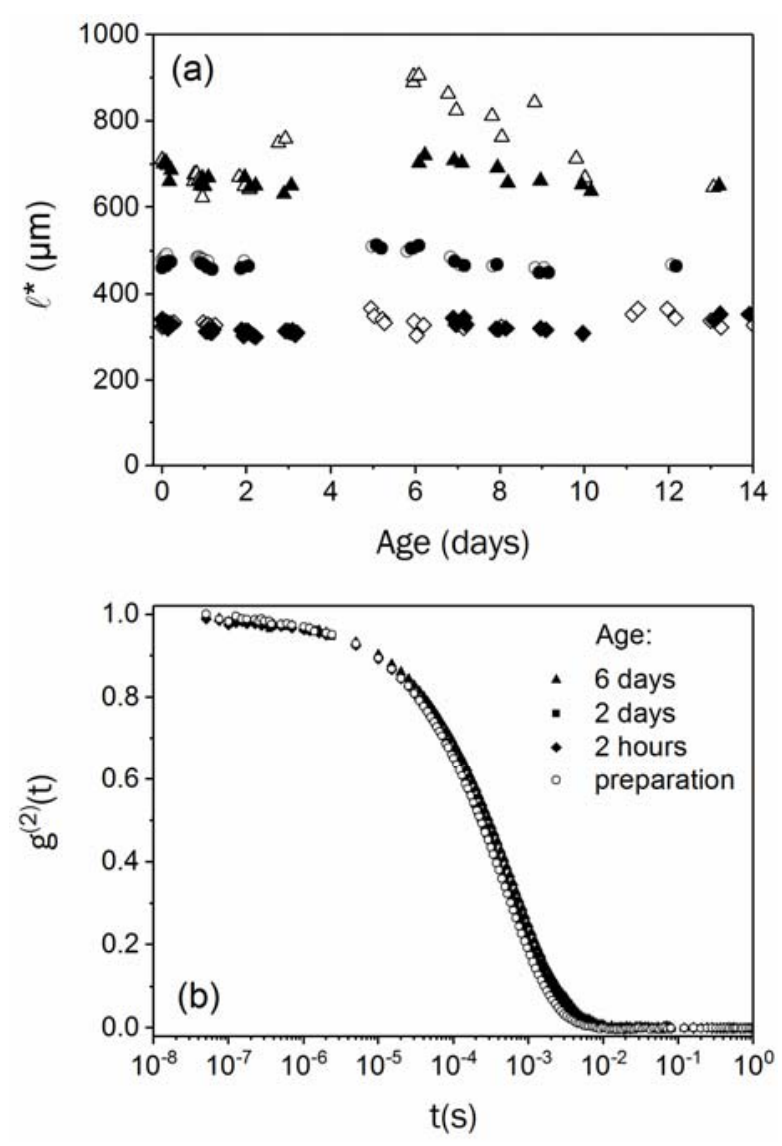

Figure 5. (a) time evolution of the transport mean free path values of emulsion gels (full symbols) and capsules (open symbols) prepared with $\mathrm{PM}(\mathrm{B}, 16)$ at $C=0.02 \mathrm{~g} / \mathrm{g}$ $(\diamond, \diamond), 0.033 \mathrm{~g} / \mathrm{g}(\bullet, \bigcirc)$, and $\mathrm{PM}(\mathrm{R}, 12)$ at $C=0.033$ $\mathrm{g} / \mathrm{g}(\boldsymbol{\Lambda}, \triangle)$. (b) intensity correlation functions at different times after preparation for a capsule suspension prepared with $\operatorname{PM}(\mathrm{B}, 16)$ at $C=0.02 \mathrm{~g} / \mathrm{g}$. The dispersed phase in (a) and (b) is silicon oil ( $\phi=0.40 ; \eta_{\mathrm{d}}=0.52$ Pa.s).

\section{DISCUSSION}

Correlation between particle size and viscoelastic properties of copolymer solutions. The prediction of droplet size in emulsions subjected to shear flow has attracted a lot of attention in the literature in relation with the fabrication of calibrated emulsions. A significant amount of literature describes the deformation and breakup of droplets when both the continuous and the dispersed phases are Newtonian fluids. The pioneering work of Taylor on the single drop problem ${ }^{40,41}$ has been expanded along various directions both theoretically and experimental$1 y^{36,39,42,43}$ Put it simply, the dynamics of single drops in shear flow is controlled by the capillary number which represents the ratio of the shear stress experienced by the droplets, $\eta_{c} \dot{\gamma}$, to the interfacial stress, $2 \Gamma / D$, where $D$ is the particle diameter. The size of Newtonian droplets sheared in a Newtonian matrix is expected to vary like the inverse of the shear rate according to the correlation: $2 \Gamma / D=$ $f\left(\eta_{c} \dot{\gamma}\right)$. This is in agreement with the result depicted in Figure $3 b$ for the aqueous solution of surfactant $S(B, 16)$. However in Fig. 3b-c, the data obtained for copolymer solutions clearly reveal strong deviations from this simple prediction. To go further it is necessary to take into account the viscoelastic properties of the continuous phase.

Two main phenomena have been reported. First it has been observed that oil in water emulsions are monodisperse when the continuous phase is viscoelastic. ${ }^{44,45}$ This has been later attributed to a modification of the break up mechanism responsible for droplet formation. ${ }^{46}$ It is likely that here similar mechanisms contribute to narrow the droplet size distribution as shown in the previous section. A second set of results concern the influence of the matrix elasticity on the generation and subsequent dynamics of droplets. Although the subject has long been controversial there is now evidence that normal stresses tend to stabilize droplets and delay fracture. ${ }^{47-54}$ Since the normal stress differences increase with the shear rate (Figure S2), the result is a slower decrease of the droplet size with the shear rate and polymer concentration than in the Newtonian case (Figure 3). A complete theory incorporating the action of the normal stress difference is beyond the scope of this paper. In the following we propose an empirical correlation which enables us to rationalize the droplet size of emulsion gels and capsules prepared over a wide range of experimental conditions, copolymer architecture and concentration, and dispersed phase viscosity.

We consider that the first normal stress difference $N_{1}$ brings the dominant contribution to the matrix elasticity and that its stabilizing effect can be expressed as an effective stress which is the sum of the interfacial pressure $2 \Gamma / D$ and $N_{1}$. This leads us to generalize the correlation

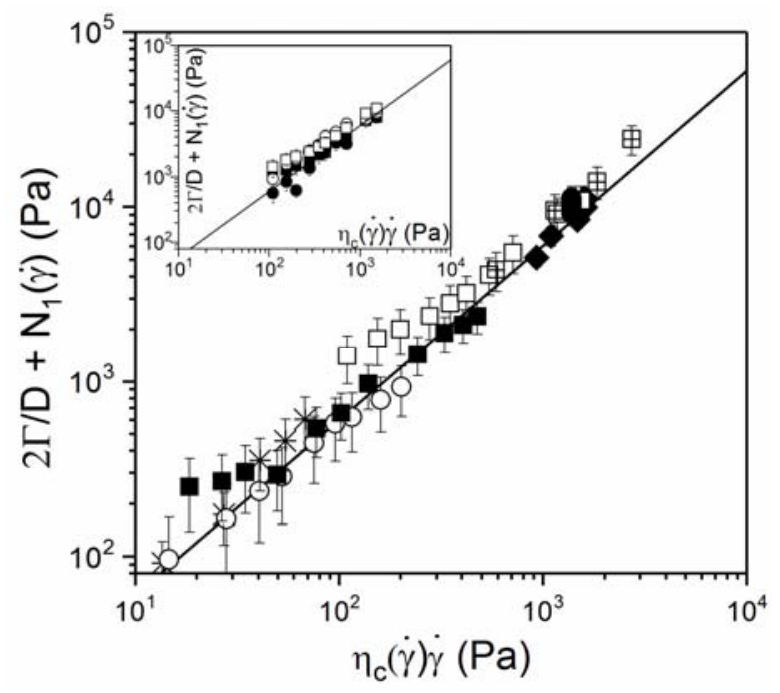

Figure 6. Correlation between the shear stress and the effective interfacial stress on the droplet. The data are for different copolymers at $C=0.033 \mathrm{~g} / \mathrm{g} \quad(\bigcirc: \mathrm{P} ; \mathbf{\square}$ : $\mathrm{PM}(\mathrm{R}, 12) ; \diamond \mathrm{PM}(\mathrm{L}, 12) ; 0: \mathrm{PM}(\mathrm{B}, 16))$ and $\mathrm{PM}(\mathrm{R}, 12)$ at $C=0.033$ (口), $0.066(\square)$, and $0.10 \mathrm{~g} / \mathrm{g} \quad$ (田); *: surfactant $\mathrm{S}(\mathrm{B}, 16)$ at $C=0.17 \mathrm{~g} / \mathrm{g}$. The inset shows the correlation for $\operatorname{PM}(\mathrm{R}, 12)$ at $C=0.066 \mathrm{~g} / \mathrm{g}$ for $\eta_{\mathrm{d}}=0.02$ $(\square), 0.52(\triangle), 1.06(\diamond)$, and $6.9(\bullet)$ Pa.s. (see also Figure S4). The solid line is the correlation for Newtonian liquids. $^{36}$ 


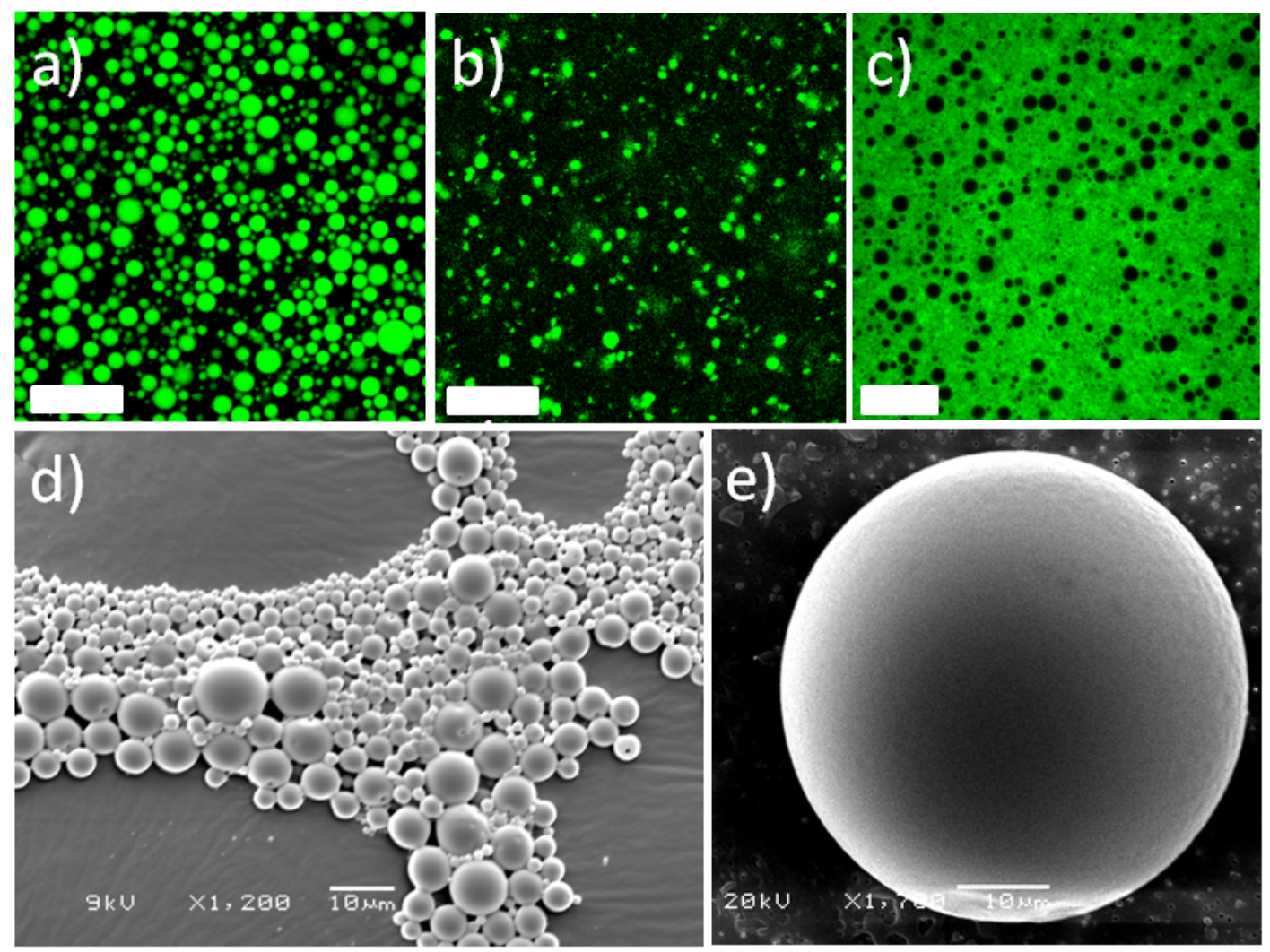

Figure 7: Confocal microscopy images of capsule dispersions made from: a) commercial olive oil ( $\eta_{\mathrm{d}} \cong 85$ mPa.s; $\left.\left.\phi_{\mathrm{d}}=0.40\right) ; \mathrm{b}\right)$ citronellal $\left.\left(\eta_{\mathrm{d}} \cong 1.5 \mathrm{mPa} . \mathrm{s} ; \phi_{\mathrm{d}}=0.15\right) ; \mathrm{c}\right)$ Esterol A $\left(\eta_{\mathrm{d}} \cong 7 \mathrm{mPa} . \mathrm{s} ; \phi_{\mathrm{d}}=0.40\right)$; dyes are Nile Red in a) and b), Rhodamine B in c); scale bar: $10 \mu \mathrm{m}$. d) and e) are scanning electronic micrographs of alkyd paint capsules ( $\eta_{\mathrm{d}} \cong 20$ $\mathrm{mPa} . \mathrm{s})$. The polymer is $\operatorname{PM}(\mathrm{B}, 16)$ at $C=0.033 \mathrm{~g} / \mathrm{g}$.

existing in the Newtonian case by plotting $2 \Gamma / D+N_{1}$ against $\eta_{c} \dot{\gamma}$. It is important to note that both the viscosity $\eta_{c}$ and the first normal stress difference $N_{1}$ of the continuous phase are shear rate dependent. Figure 6 shows that this representation collapses the data obtained for the different copolymers at all concentrations, and different viscosities of the continuous phase. The correlation also matches data for the low molecular weight surfactant $\mathrm{S}(\mathrm{B}, 16)$ and is in agreement with the correlation expected for Newtonian matrices $\left(N_{1}=0\right)$. Hence it appears that the empirical effective stress $2 \Gamma / D+N_{1}$ qualitatively accounts for the stabilizing effect associated with the first normal stress difference. Clearly this correlation needs more elaboration and should be put on a rigorous basis.

Critical evaluation of copolymer performance in encapsulation. Our technology is based on a unique combination of properties. At oil/water interfaces, the hydrophobic groups $\mathrm{X}$ place themselves inside the oil phase whereas the hydrophilic polyoxyethylene spacers and the backbones stay in the aqueous phase. The copolymers thus play the role of macromolecular surfactants which reduce the interfacial energy. Because of the comb-like structure of the copolymers, several hydrophobic groups per chain $(\cong 25)$ are solubilized in the oil phase, resulting in strong attachment and providing the long-term stability demonstrated in Figure 5. The hydrophobicity of the polyelectrolyte backbone provides $\mathrm{pH}$-responsiveness: ${ }^{.5}$ the copolymers switch from a fully extended conformation in basic solutions where the carboxylic functions of the MAA units are ionized to a collapsed conformation at low $\mathrm{pH}$ where they are protonated. PH-responsiveness allows us to precipitate the polymer adsorbed at oil-water interfaces into an insoluble polymer layer which wraps the liquid core of the capsules. Finally, the groups $\mathrm{X}$ associate into micelles in the continuous phase, which connect the polymer chains into a dynamic three-dimensional polymeric network responsible for viscoelastic properties.

The viscoelasticity of the continuous phase controls the size of the emulsion gel droplets. Figure 6 allows us to assess the relative advantages and drawbacks to the different copolymers available to encapsulate hydrophobic substances. The left hand-side of the diagram is representative of dispersions with large droplet sizes which are prepared using a low viscosity continuous phase and/or low shear rates. The right hand-side is indicative of small droplets prepared with a highly viscous matrix and/or large shear rates. Actually the performance of a copolymer results from a compromise between the shear viscosity of the continuous phase which favors small droplets and its elasticity which stabilizes droplets against break up. For a given architecture, the shear viscosity $\eta_{\mathrm{c}}$ and the normal stress difference $N_{1}$ of copolymer solutions increase with the number of carbons in the hydrophobic groups, $m$ (Figure S2). For a given $m, \eta_{\mathrm{c}}$ and $N_{1}$ vary with the architecture 
according to the rule linear $>$ branched $>$ ramified (Figure S2). Small droplets can thus be obtained at large shear rates with $\mathrm{PM}(\mathrm{R}, 12)$ at large concentration or equivalently with $\operatorname{PM}(\mathrm{B}, 16)$ at much lower concentration. However in the latter case, this is at the expense of large normal stress differences. Ramified copolymers $\mathrm{PM}(\mathrm{R}, 12)$ are thus well suited for applications where large droplets or large polymer concentrations are acceptable. Small droplets can be obtained at low polymer content and low shear intensity using copolymers providing highly viscoelastic properties like $\operatorname{PM}(\mathrm{B}, 16)$ or $\mathrm{PM}(\mathrm{L}, 16)$.

Versatility of the technology. The technology described in this paper can be applied to encapsulate a great variety of hydrophobic substances. To demonstrate its versatility, we encapsulated various hydrophobic substances commonly used in applications, which have different polarities. Figure 7 shows encapsulations of a commercial olive oil, a low-viscosity antifungal and insecticide oil (Citronellal), a lubricant (Esterol A), and an alkyd paint resin after evaporation of the aqueous continuous phase. We observe that all these liquids are dispersed in well-defined spherical particles irrespectively of their physical and chemical properties. In practical applications, the oil can be a chemical to encapsulate or simply a carrier for soluble hydrophobic active substances.

\section{CONCLUSIONS}

We have described a simple, versatile and robust technology to encapsulate a great variety of hydrophobic substances in a controlled and rational way. It exploits the interfacial properties, viscoelasticity, and $\mathrm{pH}$ responsiveness of functional amphiphilic copolymers in aqueous solutions. The technology is versatile in the sense that the same copolymer can be used to encapsulate hydrophobic liquids which differ in terms of chemical composition, polarity, viscosity, and volume fraction. Moreover the capsules prepared by this technique can be combined with other rheological additives while keeping their stability. Thickening agents like Carbopol or other microgel based additives can be added to the dispersions to prevent creaming without loss of properties. The negative charges borne by the capsules could be exploited to initiate multilayer growth with cationic polymers.

The size of the emulsion droplets and capsules is set by the emulsification step and depends on several experimental parameters which have been addressed in detail: the shear rate applied during emulsification, the copolymer concentration, the viscosities of the dispersed and continuous phases, the volume fraction of the dispersed phase, the interfacial tension between the polymer solution and the hydrophobic liquid. These parameters are not independent and a simple correlation between the effective interfacial stress $\mathbf{2} \boldsymbol{\Gamma} / \boldsymbol{D}+\boldsymbol{N}_{\mathbf{1}}$ and the shear stress in the continuous phase $\boldsymbol{\eta}_{\mathbf{c}}(\dot{\boldsymbol{\gamma}}) \dot{\boldsymbol{\gamma}}$ collapses the data obtained for the different copolymers when the concentration, the volume fraction, and the continuous phase viscosity are systematically varied. This correlation provides useful guides to select the appropriate copolymer for specific applications. By tuning the composition and the architecture of the copolymers, the technology is thus able to comply with specific needs in terms of particle morphology and processing conditions. The method is compatible with various processing techniques such as membrane emulsification or microfluidic techniques. Further developments and industrial scale-up are under progress to apply the potentialities of the technique to a wide range of applications. From a fundamental perspective, this work points to the importance of the viscoelasticity of the continuous phase and highlights the specificities of emulsification in viscoelastic media. A microscopic theory is highly desirable to build a predictive framework relating the particle size and morphology to the different parameters of the formulation.

\section{ASSOCIATED CONTENT}

\section{Supporting information}

Figure S1: Interfacial properties of copolymer solutions Figure S2: Non linear rheology of copolymer solutions Figure S3: Influence of the volume fraction of the dispersed phase on particle size

Figure S4: Influence of the viscosity of the dispersed phase on particle size

Figure S5: Shelf-life stability of emulsions

\section{AUTHOR INFORMATION}

\section{Corresponding Author}

* E-mail: michel.cloitre@espci.fr

Author Contributions This work is part of the $\mathrm{PhD}$ thesis of Clémentine Locatelli-Champagne prepared at ESPCI under the supervision of Michel Cloitre and in collaboration with Jean-Marc Suau and Olivier Gueret at Coatex. Charlotte Pellet built the DWS set-up and characterized the long term stability of emulsion gels and capsules.

\section{ACKNOWLEDGMENTS}

Michel Cloitre and Clémentine Locatelli-Champagne are indebted to R. T. Bonnecaze for enlightening discussions concerning the role of normal stress differences in emulsification.

\section{REFERENCES}

(1) Guzey D.; McClements, D. J. Formation, stability and properties of multilayer emulsions for application in the food industry. Adv. Colloid Interface Sci. 2006, 128-130, 227-248.

(2) Vemmer, M.; Patel, A. V. Review of encapsulation methods suitable for microbial biological control agents. Biological Control 2013, 67, 380-389.

(3) Wang, Y.; Byrne, J. D.; Napier, M. E. ; DeSimone, M. E. Engineering nanomedicines using stimuli-responsive biomaterials. Adv. Drug Deliv. Rev. 2012, 64, 1021-1030.

(4) De Cock, L. J.; De Koker, S.; De Geest, B. G.; Grooten, J; Vervaet, C.; Remon, J. P.; Sukhorukov, G. B.; Antipina, M. N. Polymeric multilayer capsules in drug delivery. Angew. Chem. Int. Ed. 2010, 49, 6954-4973. 
(5) Soussan, E.; Cassel, S.; Blanzat, M.; Rico-Lattes, I. Drug delivery by soft matter: matrix and vesicular carriers. Angew. Chem. Int. Ed. 2009, 48, 274-288.

(6) Yow, H. N.; Routh, A; F. Formation of liquid core-polymer shell microcapsules. Soft Matter 2006, 2, 940-949.

(7) Caruso, F.; Caruso, R. A.; Möhwald, H. Nanoengineering of inorganic and hybrid hollow spheres by colloidal templating. Science 1998, 282, 1111-1114.

(8) Donath, E.; Sukhorukov, G. B.; Caruso, F.; Davis, S. A.; Möhwald, H. Novel hollow polymer shells by colloid-templated assembly of polyelectrolytes. Angew. Chem. Int. Ed. 1998, 37, 22012205.

(9) Grigoriev, O.; Bukreeva, T.; Möhwald, H.; Shchukin, D. G.; New method for fabrication of loaded micro- and nanocontainers: emulsion encapsulation by polyelectrolyte layer-by-layer deposition on the liquid core. Langmuir 2008, 24, 999-1004.

(10) Priest, C.; Quinn, A.; Postma, A.; Zelikin, A. N.; Ralston, J; Caruso, F. Microfluidic polymer multilayer adsorption on liquid crystal droplets for microcapsule synthesis. Lab Chip 2008, 8, 21822187.

(11) Le Tirilly, S.; Tregouët, C.; Bône, S.; Geffroy, C.; Fuller, G.; Pantoustier, N.; Perrin, P.; Monteux, C. Interplay of hydrogen bonding and hydrophobic interactions to control the mechanical properties of polymer multilayers at the oil-water interface. ACS Macro Lett. 2015, 4, 25-29.

(12) Sivakumar, S.; Bansal, V.; Cortez, C.; Chong, S.-F.; Zelikin, F. Caruso, A. N. Degradable, Surfactant-free, monodisperse polymerencapsulated emulsions as anticancer drug carriers. Adv. Mater. 2009, $21,1820-1824$.

(13) Dobashi, T.; Furukawa, T.; Narita, T. ; Shimofure, S.; Ichikawa, K.; Chu, B. Determination of the swelling ratio of Poly(Urea urethane) microcapsules by single-particle light scattering. Langmuir 2001, 17, 4525-4528.

(14) Monteillet, H.; Hagemans, F.; Sprakel, J. Charge-driven coassembly of polyelectrolytes across oil-water interfaces. Soft Matter 2013, 9, 11270-11275.

(15) Kaufman, G.; Boltyanskiy, R.; Nejati, S.; Thiam, A. R.; Loewenberg, M.; Dufresne, E. R.; Osuji, C. O. Single-step microfluidic fabrication of soft monodisperse polyelectrolyte microcapsules by interfacial complexation. Lab. Chip 2014, 14, 3494-3497.

(16) Kim, M.; Yeo, S. J.; Highley, C. B.; Burdick, J. A.; Yoo, P. J.; Doh, J.; Lee, D. One-Step Generation of Multifunctional Polyelectrolyte Microcapsules via Nanoscale Interfacial Complexation in Emulsion (NICE). ACS Nano 2015, 9, 8269-8278.

(17) Cui, J.; Wang, Y.; Postmo, A.; Hao, J.; Hosta-Rigau, A.; Caruso, F. Monodisperse polymer capsules: tailoring size, shell thickness, and hydrophobic cargo loading via emulsion templating. Adv. Funct. Mat. 2010, 20, 1625-1631.

(18) Vladisavljević, G. T.; Duncanson, W. J.; H. C. Shum, D. A. Weitz. Emulsion templating of Poly(lactic acid) particles: droplet formation behavior. Langmuir 2012, 28, 12948-12954.

(19) Patra, D.; Ozdemir, F.; Miranda, O. R.; Samanta, B.; Sanyal, A.; Rotello, V. M. Formation and size tuning of colloidal microcapsules via host-guest molecular recognition at the liquid-liquid interface. Langmuir 2009, 25, 13852-13854.

(20) Zhang, J.; Coulston, R. J.; Jones, S. T.; Geng, J.; Scherman, O. A.; C. Abell. One-Step fabrication of supramolecular microcapsules from microfluidic droplets. Science 2012, 335, 690-694.

(21) Loxley, A.; Vincent, B. Preparation of Poly(methyl methacrylate) microcapsules with liquid cores. J. Colloid Interface Sci. 1998, 208, 49-62.

(22) Dowding, P. J.; Atkin, R.; Vincent, B.; Bouillot, P. Oil corepolymer shell microcapsules prepared by internal phase separation from emulsion droplets. I. Characterization and release rates for microcapsules with Polystyrene shells. Langmuir 2004, 20, 1137411379.

(23) Yuan, Q.; Williams, R. A.; Biggs, S. Surfactant selection for accurate size control of microcapsules using membrane emulsification. Colloids Surf. A 2009, 347, 97-103.
(24) Choi, C.-H.; Weitz, D.A.; Lee, C.-S. One step formation of controllable complex emulsions: from functional particles to simultaneous encapsulation of hydrophilic and hydrophobic agents into desired position. Adv. Mat. 2013, 25, 2536-2541.

(25) Datta, S. S.; Abbaspourrad, A.; Amstad, E.; Fan, J.; Kim, S.-H.; Romanowsky, M.; Shum, H. C.; Sun, B.; Utada, A. S.; Windbergs, M.; Zhou, S.; Weitz, D. A. 25th anniversary article: double emulsion templated solid microcapsules: mechanics and controlled release. Adv. Mater. 2014, 26, 2205-2218.

(26) Tadros, T. Polymeric surfactants in disperse systems. Adv. Colloid Interface Sci. 2009, 147, 281-299.

(27) Discher, B. M.; Won, Y.-Y.; Ege, D. S.; Lee, J. C-M.; Bates, F. S.; Discher, D. E.; Hammer, D. A. Polymersomes: tough vesicles made from diblock copolymers. Science 1999, 284 1143-1146.

(28) Mathur, A. M.; Drescher, B.; Scranton, A. B.; Klier, J. Polymeric emulsifiers based on reversible formation of hydrophobic units. Nature 1998, 392, 367-370.

(29) Weaver, J. V. M.; Rannard, S. P.; Cooper, A. I. Polymermediated hierarchical and reversible emulsion droplet assembly. Angew. Chem. Int. Ed. 2009, 48, 2131-2134.

(30) Li, W.; Yu, Y.; Lamson, M.; Silverstein, M. S.; Tilton, R. D.; Matyjaszewski, K. PEO-based star copolymers as stabilizers for water-in-oil or oil-in-water emulsions. Macromolecules 2012, 45, 9419-9426.

(31) Berry, J. D.; Neeson, M. J.; Dagastine, R. D.; Chan, D.Y.C.; Tabor, R. F. Measurement of surface and interfacial tension using pendant drop tensiometry. J. Colloid Interface Sci. 2015, 454, 226237.

(32) Kumacheva, E., Rharbi, Y., Winnik, M.A., Guo, L., Tam, K.C., Jenkins, R.D., Fluorescence studies of an alkali-swellable associative polymer in aqueous solution. Langmuir 1997, 13, 182.

(33) Locatelli-Champagne, C.; Cloitre, M. Monitoring mesoglobules formation in PNIPAm solutions using Nile Red solvatochromism, Colloid Polym. Sci. 2003, 291, 2911-2916

(34) Tirtaatmadja, V.; Tam, K. C.; Jenkins, R. D. Rheological properties of model alkali-soluble associative (HASE) polymers: effect of varying hydrophobe chain length. Macromolecules 1997, 30, 32713282.

(35) Corte, L.; Leibler, L. Analysis of polymer blend morphologies from transmission electron micrographs. Polymer 2005, 46, 63606368.

(36) Schmitt, V.; Leal-Calderon, F.; Bibette, J. Preparation of monodisperse particles and emulsions by controlled shear. Top. Curr. Chem. 2003, 227, 195-215.

(37) Monti, F.; Fu, S.-Y.; Iliopoulos, I.; Cloitre, M. Doubly responsive polymer-microgel composites: rheology and structure. Langmuir 2008, 24, 11474-11482.

(38) Rojas-Ochoa, L. F.; Romer, S.; Scheffold, F.; Schurtenberger, P. Diffusing-wave spectroscopy and small-angle neutron scattering from concentrated colloidal suspensions. Phys. Rev. E 2002, 65, 051403.

(39) Karam, H. J.; Bellinger, J. C. Deformation and breakup of liquid droplets in a simple shear field. I\&EC Fundamentals 1968, 7, 576581.

(40) Taylor, G. I. The viscosity of a fluid containing small drops of another fluid. Proc. R. Soc. London A 1932, 138, 41-48.

(41) Taylor, G. I. The formation of emulsions in definable fields of flow. Proc. R. Soc. London A 1934, 146, 501-523.

(42) Grace, H. P. Dispersion phenomena in high viscosity immiscible fluid systems and application of static mixers as dispersion devices in such systems. Chemical Engineering Communications 1982, 14, 225277.

(43) Stone, H. A. Dynamics of drop deformation and breakup in viscous fluids. Annu. Rev. Fluid Mech. 1994, 26, 65-102.

(44) Mason, T. G., Bibette, J. Emulsification in viscoelastic media. Phys. Rev. Lett. 1996, 77, 3481-3484.

(45) Mason, T. G.; Bibette, J. Shear rupturing of droplets in complex fluids. Langmuir 1997, 13, 4600-4613.

(46) Zhao, X.; Goveas, J. L. Size selection in viscoelastic emulsions under shear. Langmuir 2001, 17, 3788-3791. 
(47) Mighri, F.; Carreau, P. J.; Ajji A. Influence of weak elasticity of dispersed phase on droplet behavior in sheared polybutadiene/poly(dimethyl siloxane) blends. J. Rheol. 1998, 42, $1477-1490$.

(48) Maffettone, P. L.; Greco, F. Ellipsoidal drop model for single drop dynamics with non-Newtonian fluids. J. Rheol. 2004, 48, 83100 .

(49) Sibillo, V.; Simeone, M.; Guido, S. Break-up of a Newtonian drop in a viscoelastic matrix under simple shear flow. Rheol. Acta 2004, 43, 449-456.

(50) Minale, M. Deformation of a non-Newtonian ellipsoidal drop in a non-Newtonian matrix: extension of Maffettone-Minale model. $J$. Non-Newtonian Fluid Mech. 2004, 123, 151-160.

(51) Yu, P.; Feng, J. M.; Liu, C.; Shen, J. Viscoelastic effects on drop deformation in steady shear. J. Fluid Mech. 2005, 540, 427-437.
(52) Verhulst, K.; Moldenaers, P.; Minale, M. Drop shape dynamics of a Newtonian drop in a non-Newtonian matrix during transient and steady shear flow. J. Rheol. 2007, 51, 261-273.

(53) Aggarwal, N.; Sarkar, K. Effects of matrix viscoelasticity on viscous and viscoelastic drop deformation in a shear flow. $J$ Fluid Mech. 2008, 601, 63-84.

(54) Verhulst, K.; Cardinaels, R.; Moldenaers, P.; Renardy, Y., Afkhami S. Influence of viscoelasticity on drop deformation and orientation in shear flow. J. Non-Newtonian Fluid Mech. 2009, 156, $29-43$.

(55) Borukhov, I; Andelman, D.; Borrega, R.; Cloitre, M.; Leibler, L.; Orland, H. Phys. Chem. B 2000, 104, 11027-1034. 


\section{Versatile encapsulation technology based on tailored pH-responsive} amphiphilic polymers: emulsion gels and capsules

Clémentine Locatelli-Champagne ${ }^{1,2}$, Jean-Marc Suau ${ }^{2}$, Olivier Guerret ${ }^{2,3}$, Charlotte Pellet ${ }^{1}$, Michel Cloitre ${ }^{* 1}$

${ }^{1}$ Soft Matter and Chemistry, CNRS, ESPCI Paris, PSL Research University, 10 rue Vauquelin, 75005 Paris (France)

${ }^{2}$ Coatex SAS, 35 Rue Ampère, 69730 Genay (France)

${ }^{3}$ M2i Life Sciences, 1 Rue Royale, 92210 Saint Cloud (France)

*Corresponding author: michel.cloitre@espci.fr

\section{Interfacial properties of copolymer solutions}

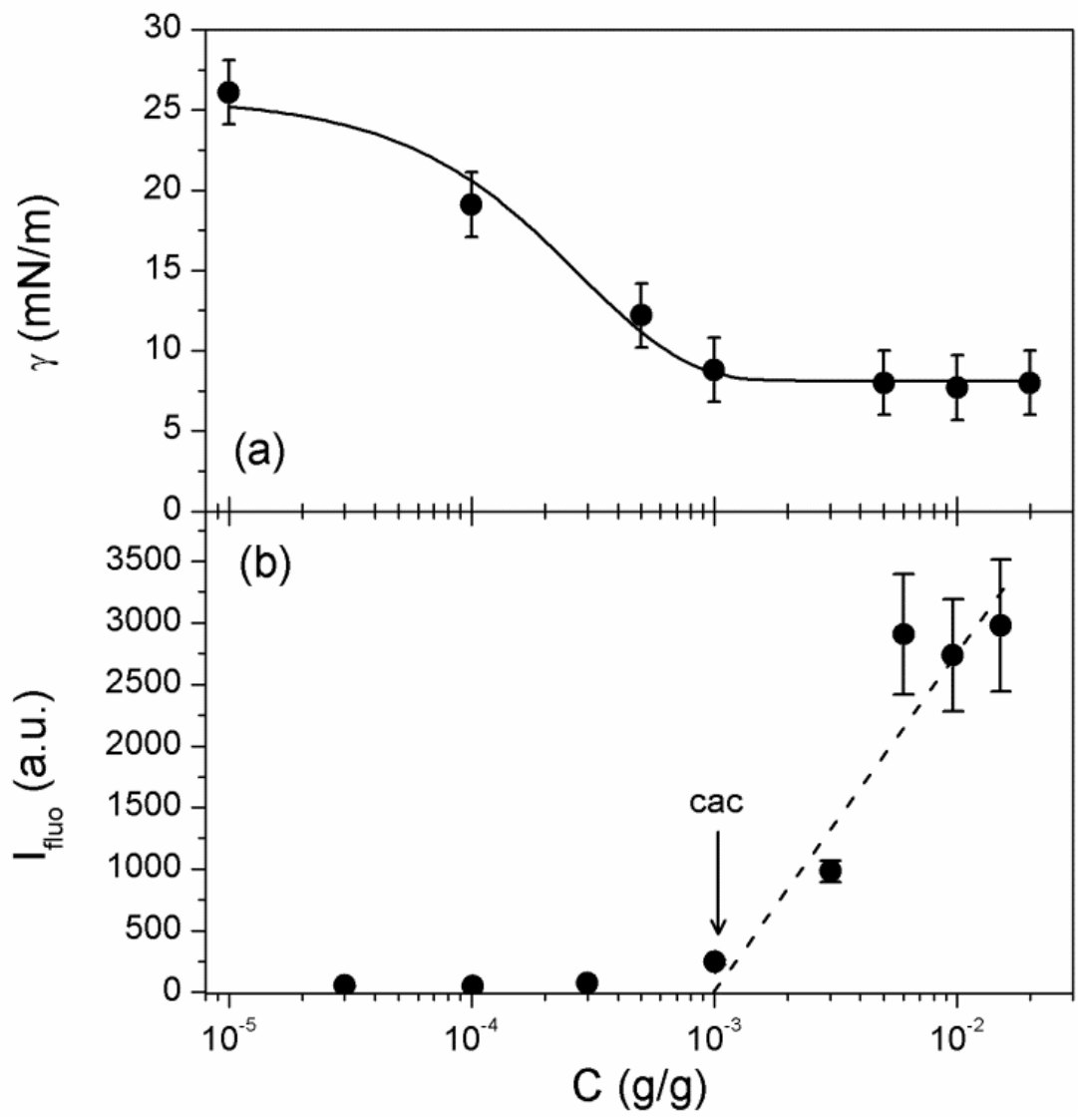

Figure S1. a) Interfacial tension between aqueous solutions of $\operatorname{PM}(B, 16)$ and silicon oil versus polymer concentration; the continuous line is an exponential fit to the experimental data. b) Fluorescence intensity of Nile Red in aqueous solutions of $\mathrm{PM}(\mathrm{B}, 16)$ and determination of the critical aggregation concentration $\left(\lambda_{\mathrm{exc}}=543 \mathrm{~nm}\right)$. 


\section{Non linear rheology of copolymer solutions}

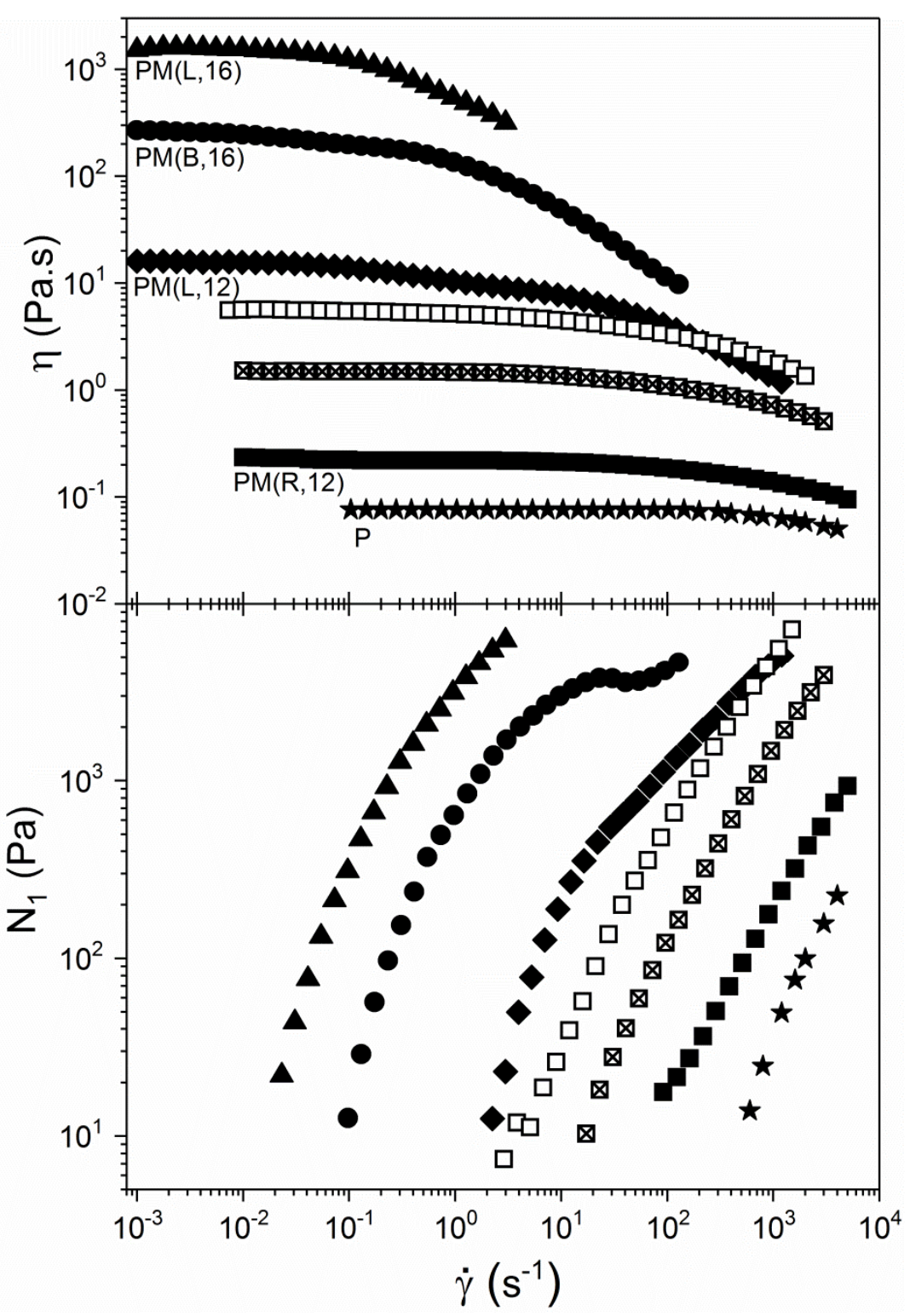

Figure S2. Steady shear viscosity and first normal stress difference of aqueous solutions of the different copolymers used in this study $\left(C=3.3 \times 10^{-2} \mathrm{~g} / \mathrm{g}\right): \mathbf{\Delta}: \operatorname{PM}(\mathrm{L}, 16) ; \mathbf{O}: \operatorname{PM}(\mathrm{B}, 16)$; ४: $\operatorname{PM}(\mathrm{L}, 12) ; \mathbf{\square}: \operatorname{PM}(\mathrm{R}, 12) ; \star:$ P. Square symbols refer to $\mathrm{PM}(\mathrm{R}, 12)$ at different concentrations: $\mathbf{\square}: C=3.3 \times 10^{-2} \mathrm{~g} / \mathrm{g} ; \bigotimes: C=6.6 \times 10^{-2} \mathrm{~g} / \mathrm{g} ; \square: C=10^{-1} \mathrm{~g} / \mathrm{g}$. 


\section{Influence of the volume fraction of the dispersed phase on particle size}

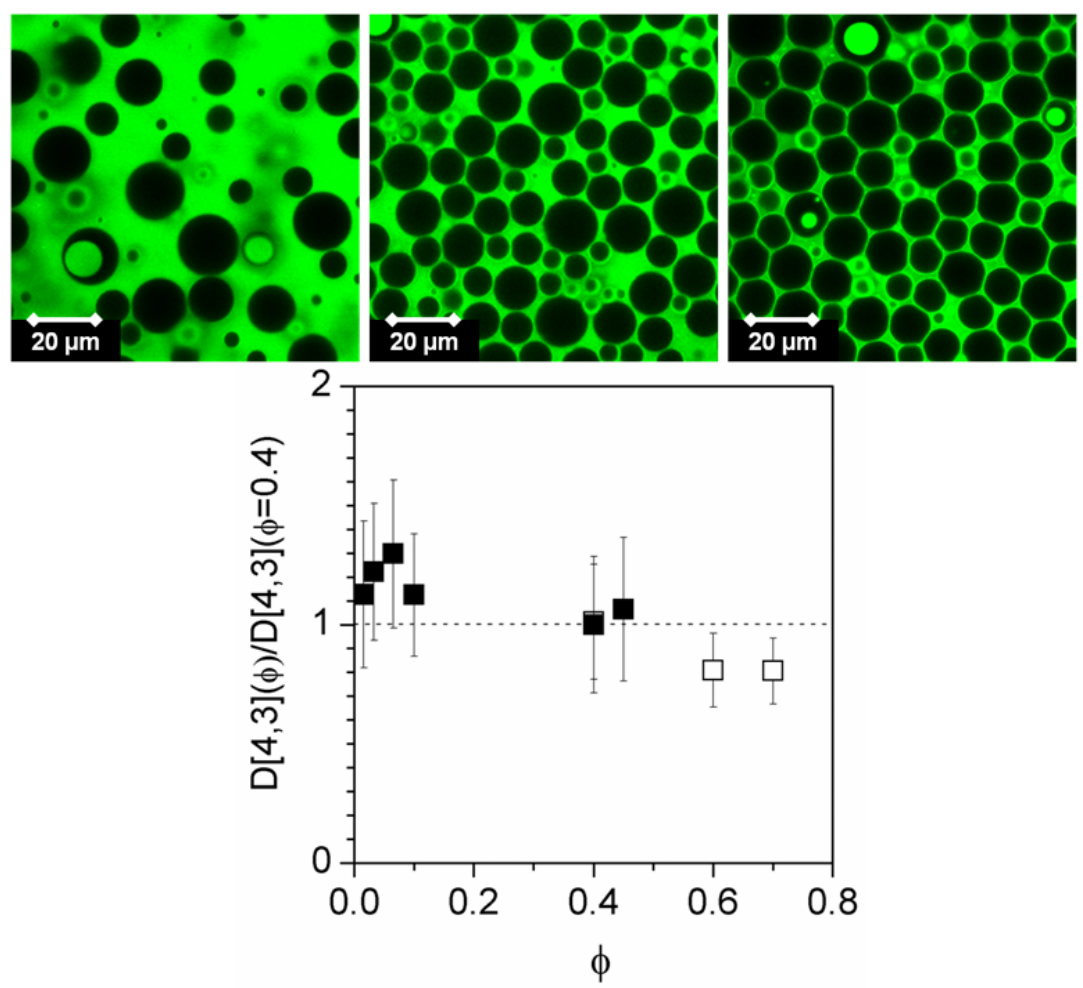

Figure S3. Top: silicone oil/water emulsion gels prepared with $\mathrm{PM}(\mathrm{R}, 12)$ at $C=0.033 \mathrm{~g} / \mathrm{g}$ for increasing oil volume fractions: $\phi=0.40,0.60$, and 0.70 ; the dye is Rhodamine $\mathrm{B}$ $\left(\lambda_{\mathrm{exc}}=514 \mathrm{~nm}\right)$. Bottom: normalized volume mean diameter versus $\phi(\boldsymbol{\square}: C=0.066 \mathrm{~g} / \mathrm{g} ; \square$ : $\mathrm{C}=0.033 \mathrm{~g} / \mathrm{g}) ; \eta_{\mathrm{d}}=0.52$ Pa.s and $\dot{\gamma}=10^{3} \mathrm{~s}^{-1}$. 


\section{Influence of the viscosity of the dispersed phase on particle size}
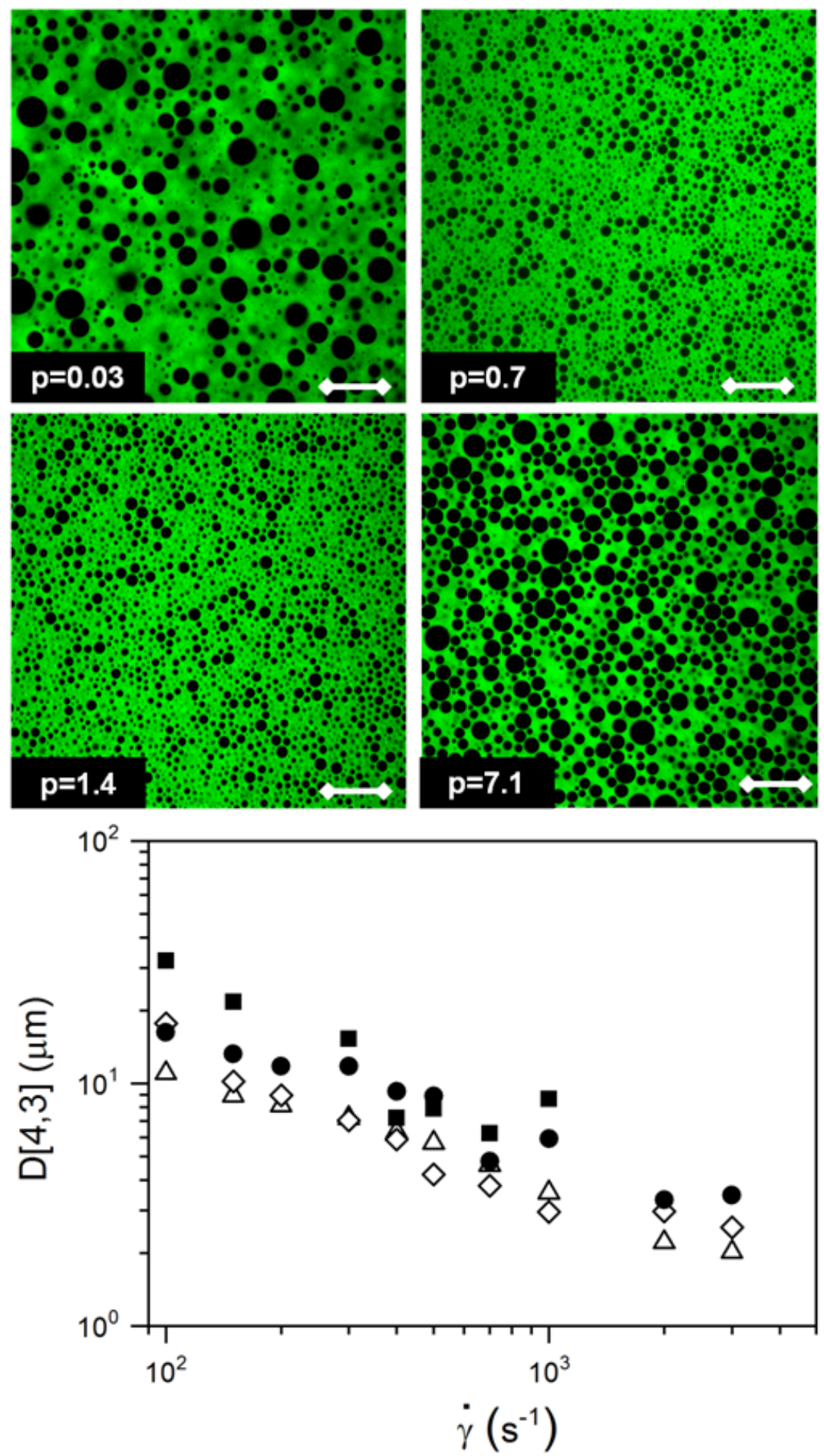

Figure S4: Top: silicone oil/water emulsion gels prepared with $\operatorname{PM}(\mathrm{R}, 12)$ at $C=0.066 \mathrm{~g} / \mathrm{g}$ for $\eta_{\mathrm{d}}=0.022,0.52,1.06$, and 6.9 Pa.s, from top to bottom; $p$ is given at the shear rate of preparation $\dot{\gamma}=10^{3} \mathrm{~s}^{-1} ; \phi=0.40$; the dye is Rhodamine B ( $\lambda_{\text {exc }}=514 \mathrm{~nm}$ ) Bottom: volume mean diameter of droplets versus shear rate for $\eta_{\mathrm{d}}=0.02(\mathbf{\square}), 0.52(\triangle), 1.06(\diamond)$, and 6.9 (๑) Pa.s. 

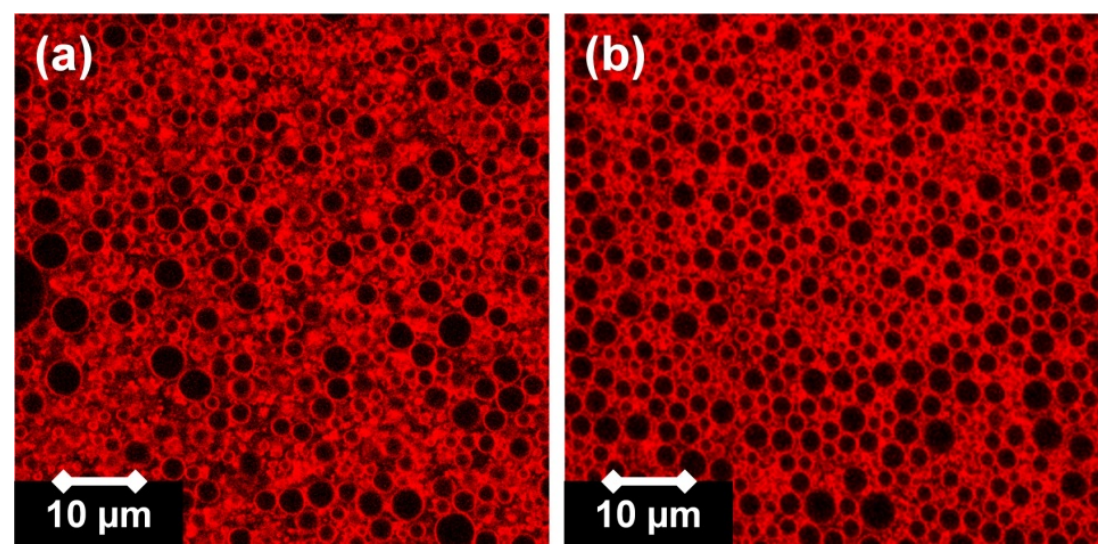

Figure S5: Confocal image of an emulsion taken just after preparation (a) and after six months (b). The dye is Nile Red ( $\left.\lambda_{\mathrm{exc}}=543 \mathrm{~nm}\right)$. 